\title{
Eficacia de un programa cognitivo-conductual para hombres maltratadores en la relación de pareja
}

\section{Effectiveness of a Cognitive-Behavioral Program for Male Batterers in the Intimate Partner Relationship}

\author{
Fredrik Lúcar Vidal ${ }^{1}$, Alberto Loharte Quintana Peña ${ }^{2}$ \\ Universidad Nacional Mayor de San Marcos
}

Recibido: $16-03-21$

Aceptado: $26-04-21$

Publicado: $18-06-21$

\section{Resumen}

La investigación tuvo como objetivo comprobar la eficacia de un programa de tratamiento cognitivo conductual con enfoque de género en hombres agresores en la relación de pareja. La muestra estuvo conformada por 48 hombres que están denunciados por violencia contra su pareja y que han sido forzados a llevar a cabo tratamiento psicológico por orden judicial. La mayoría de los cuales fueron adultos, sin antecedentes de psicopatología grave, laboralmente activos, con antecedentes de violencia en su familia de origen y sin problemas de consumo de alcohol, la mitad de los mismos con solo educación primaria y secundaria. Evaluados antes y después del tratamiento con la Escala de Tácticas de Conflictos (CTS-2), el Inventario de Pensamientos Distorsionados hacia la Mujer y la Violencia (IPMV) y la Escala de Atribución de Responsabilidad y Minimización (IRM). Se analizaron los datos con la prueba t para muestras relacionadas, reportándose el tamaño del efecto a través del coeficiente Delta de Glass. Los resultados indican que el programa del tratamiento fue eficaz en disminuir de manera significativa la frecuencia de conducta violenta, las atribuciones de responsabilidad y las distorsiones cognitivas referentes tanto a la mujer como al uso de la violencia.

Palabras clave: Pensamientos Distorsionados; Violencia contra la pareja; Atribución de Responsabilidad; Minimización; Programa cognitivo conductual.

\begin{abstract}
The purpose of the research was to verify the efficacy of a cognitive behavioral treatment program with a gender perspective in male batterers in the relationship. The sample of 48 men who were reported for violence against their intimate partner, and also were forced to undergo psychological treatment by court order. Most of them were adults, without a history of serious psychopathology, employed, with a history of violence in their family of origin

1 Docente, Universidad Nacional Mayor de San Marcos. Lima, Perú.

Autor para correspondencia: flucarv@unmsm.edu.pe ORCID: http://orcid.org/0000-0003-0949-859X

2 Docente, Universidad Nacional Mayor de San Marcos. Lima, Perú.

E-mail: aquintanap@unmsm.edu.pe ORCID: https://orcid.org/0000-0003-4305-137X
\end{abstract}

(C) Los autores. Este artículo es publicado por la Revista de Investigación en Psicología de la Facultad de Psicología, Universidad Nacional Mayor de San Marcos. Este es un artículo de acceso abierto, distribuido bajo los términos de la licencia Creative Commons Atribución 4.0 Internacional (CC BY 4.0) [https://creativecommons.org/licenses/by/4.0/deed.es] que permite el uso, distribución y reproducción en cualquier medio, siempre que la obra original sea debidamente citada de su fuente original. 
no alcohol consumption problems, half of them with only primary and secondary education. Assessed before and after treatment with the Conflict Tactics Scale (CTS-2), the Inventory of Distorted Thoughts towards Women and Violence (IPMV) and the Attribution of Responsibility and Minimization Scale (IRM). The data were analyzed with the t-test for related samples, and the effect size measurements were reported using the Delta coefficient of Glass. Therefore, the results show that the treatment program was effective in significantly reducing the frequency of violent behavior, attributions of responsibility, and the cognitive distortions regarding women and violence against women.

Keywords: Cognitive distortions; Violence against intimate partner; Attributions of responsibility; Minimization; Cognitive behavioral program.

Es crucial reconocer que las situaciones en las que la mujer ha sido víctima de abuso psicológico físico y/o sexual, con toda probabilidad, han existido siempre, aunque por supuesto con distintos grados de intensidad. Sin embargo, es reciente el reconocimiento de que estas situaciones han significado un atentado a sus derechos más fundamentales y, a la vez, un serio problema personal, familiar y social. Esto se puede comprobar al revisar que en la legislación de diversos países aparece como un problema recién a partir de la segunda mitad del siglo XX, además, ha sido recién en las últimas décadas del siglo pasado cuando se ha evidenciado un interés científico por estudiar y comprender este fenómeno (Menéndez et al., 2013).

Casi un tercio de mujeres en todo el mundo han mantenido una relación de pareja en la que han sido víctimas de violencia física y/o sexual por parte de aque1la (OMS, 2013, 2016). En algunas regiones, la cifra llega hasta el 38\%. Se ha llegado a considerar incluso que este tipo de violencia daña más a las mujeres que la combinación de asaltos y violaciones y accidentes de automóvil (Conchell, 2011).

Cuando la OMS realizó su Estudio multipaís sobre salud de la mujer y violencia doméstica contra la mujer (García-Moreno et al., 2005) se reportaron índices de prevalencia de violencia física y sexual contra las mujeres en varios países. Se destacaron las diferencias entre los países y se encontró que las tasas de violencia física o sexual infligida por una pareja oscilaron entre $15 \%$ en el Japón y cerca de $70 \%$ en Etiopía y Perú. Al mismo tiempo, la mayoría de los países comunicaron tasas entre $29 \%$ y $62 \%$, lo que nos indica que los índices de violencia contra la mujer por parte de la pareja en el Perú son elevados comparados con los reportados a nivel mundial. A pesar de un ligero descenso, durante la última década, las estadísticas colocan al Perú como un país con un índice alto de violencia contra la mujer que se corrobora al revisar la Encuesta Demográfica y de Salud Familiar (INEI, 2019), que muestra que a nivel nacional, el 57,7\% de las mujeres que tienen o tuvieron alguna vez una relación de pareja sufrieron algún tipo de violencia por parte del esposo o compañero, reduciéndose en 10,5 puntos porcentuales con relación al año $2016(68,2 \%)$ y 16,8 con relación al año 2012 (74,1\%). Entre las formas 
de violencia, destaca la violencia psicológica y/o verbal $(52,8 \%)$, la violencia física $(29,5 \%)$ y la violencia sexual $(7,1 \%)$.

Con estos datos se puede concluir que la violencia en la relación de pareja, por lo menos en el Perú, es un problema social y de salud pública muy importante, tanto por su elevada incidencia cuanto por la gravedad de las consecuencias negativas, físicas y psicológicas para las víctimas y sus familiares. Por ello, luego de 22 años de vigencia de la Ley 26260, "Ley de protección frente a la violencia familiar", el 24 de noviembre de 2015 entró en vigencia la nueva "Ley para prevenir, sancionar y erradicar la violencia contra las mujeres y los integrantes del grupo familiar, Ley 30364 (2015), teniendo esta última mayor alcance y especificidad que la anterior. Justamente una de las novedades fue la inclusión de un capítulo específico para la reeducación de personas agresoras. Esta norma ha diferenciado los servicios de reeducación de dos formas: a) tratamiento penitenciario para la reinserción social de las personas agresoras privadas de su libertad, y b) tratamiento para personas agresoras en medio libre. Es la segunda forma de tratamiento la que tiene relevancia para los fines de esta investigación, ya que se tratan de programas a los que están obligados a acudir personas que no han sido privadas de su libertad. Según dicta la ley, el juez puede imponer al agresor tratamiento especializado en violencia a través de la asistencia a terapias sobre violencia contra las mujeres y los integrantes del grupo familiar. Esta medida puede aplicarse desde el inicio del proceso judicial y el sometimiento a este tipo de tratamiento es considerado como regla de conducta, sin perjuicio de la sanción penal que corresponda.

En ese sentido, vale la pena poner de relieve que el tratamiento psicológico para el agresor es considerado una manera indirecta de proteger a la víctima, sin menoscabo de las medidas de protección directas que se dicten para ellas. No es suficiente con atender sólo a la víctima; es de suma importancia modificar el comportamiento del hombre que ha maltratado para que no vuelva a maltratar a esa mujer en el caso de que sigan conviviendo juntos, y en el caso de que no continúen con la relación de pareja, que ninguna otra mujer pueda ser una víctima potencial de él. Los programas terapéuticos enfocados en la figura del agresor tienen como objetivo básico y principal la prevención de la reincidencia del maltrato a la mujer dentro del ámbito familiar. Así, frente a posicionamientos más bien simplistas, el tratamiento psicológico dirigido a maltratadores de violencia de género no es tanto una medida alternativa a las medidas judiciales, sino más bien una intervención complementaria.

Estos programas se fundamentan en la constatación de que existe un conjunto de factores de riesgo relacionados con la violencia contra la pareja que se pueden modificar desde un punto de vista psicológico y educativo. Por lo tanto, aunque el agresor es el responsable de sus conductas, también muestra un conjunto de limitaciones psicológicas y formativas sobre las que se puede intervenir. Pero, sin dejar de lado que el objetivo fundamental de todos los programas de tratamiento para agresores es el control de la violencia en sus diferentes tipos. 
$\mathrm{Al}$ respecto, habría que considerar lo siguiente: el hogar, que debiera ser un lugar de protección, seguridad y afecto, paradójicamente se convierte en un entorno de impunidad para situaciones de maltrato. La familia puede constituirse en un caldo de cultivo apropiado para los maltratos repetidos y prolongados. Bajo estas circunstancias, las víctimas llegan a sentirse incapaces de escapar del control de los agresores pues muchas veces están sujetas a ellos por la fuerza física, la dependencia emocional, el aislamiento social o diferentes tipos de vínculos económicos, legales o sociales (Echeburúa et al., 2002). Podría decirse entonces que la violencia en el hogar y, más específicamente, la violencia del varón contra su pareja, está vinculada a la identidad masculina en la sociedad, conformada por un aprendizaje temprano en el hombre de ciertos valores, creencias y actitudes como son el ejercicio de la autoridad y control, la preocupación central por el éxito profesional, el ocultamiento de las emociones, entre otros (Kaufman, 1997; Seidler, 2000). Estos patrones violentos de interacción y relaciones de abuso son la consecuencia de un modelo familiar y social que las acepta como estrategias válidas para resolver conflictos.

Ahora bien, todo esto es expresado por los agresores en forma de distorsiones cognitivas relacionadas con los roles de género y la consiguiente subordinación de la mujer (misoginia), aparte de cierta legitimación de la violencia como forma adecuada para resolver conflictos (Echeburúa et al., 2003; Echeburúa y Redondo, 2010). Asimismo, una característica habitual del maltrato es la negación de esta conducta por parte del agresor. Por ello, los agresores suelen usar un conjunto de racionalizaciones para justificar su conducta violenta, evitando sentirse culpables, rechazados socialmente y emocionalmente afectados. Así, suelen utilizar estrategias como la negación y/o la minimización de la conducta violenta, la culpabilización de la víctima, la supuesta reacción de defensa propia, entre otros (Lila et al., 2008; Conchell et al., 2012).

Por todo ello, Medina (2002) consideró que un programa de tratamiento para agresores debe tener como objetivos: a) que los agresores enfrenten las consecuencias de su conducta, es decir, que se asuman la responsabilidad de los abusos cometidos, b) el control del maltrato en sus diversas formas, c) la eliminación de las distorsiones cognitivas, las racionalizaciones y justificaciones con las que intentan justificar su conducta, d) componentes preventivos orientados al manejo de la ira y el estrés, y e) la promoción del desarrollo de habilidades de comunicación.

Es necesario indicar que la evaluación de la eficacia de programas para agresores contra su pareja, se ha venido realizando en el mundo anglosajón y escandinavo (EEUU, Canadá, Australia, Suecia) desde hace treinta años (Deza y Guzmán, 2009), y recientemente en el contexto hispano (Echauri, 2010; Redondo, 2012; Pérez et al., 2013; Echauri et al., 2013; Boira et al., 2013; Arrigoni et al., 2013; De los Galanes y Tabernero, 2013; Conchell, Lila, Catalá, 2016; Carbajosa et al., 2017; Fernández-Montalvo et al., 2019; Vargas et al., 2020, Fernández-Montalvo 
et al., 2020), evidenciando cambios positivos en los agresores, es decir, tras el proceso terapéutico, estos hombres se muestran menos violentos. En ese sentido, algunos estudios han reportado la presencia de pensamientos machistas sobre la inferioridad de la mujer y sobre el uso de la violencia como estrategia válida de afrontamiento como un fenómeno observable con mucha más frecuencia en la población latinoamericana que en la norteamericana y europea (Fernández-Montalvo et al., 2011; Vargas et al., 2020), razón por la cual sería relevante comprobar la efectividad de este programa en el contexto peruano.

En suma, la intención de esta investigación fue comprobar si se producen cambios significativos tras la participación en un programa de tratamiento cognitivo conductual para hombres agresores contra su pareja, en variables relacionadas con esta violencia y abordadas de manera específica en el programa de tratamiento.

\section{Método}

\section{Diseño}

La presente investigación se clasifica dentro de los diseños cuasiexperimentales, específicamente el diseño pretest-postest (DPP), tal como lo señala Ato, López y Benavente (2013).

\section{Participantes}

Se utilizó una muestra de 119 hombres obligados a llevar a cabo terapia psicológica en medio libre por orden judicial, de los cuales nueve no cumplieron con los criterios de inclusión al programa: 1) hombre mayor de 18 años, 2) sin psicopatología grave, 3) sin déficit significativo de su capacidad física y/o cognitiva que le dificulte el cumplimiento de los objetivos del programa y 4) en caso de tener alguna adicción a drogas y/o alcohol estar, en paralelo, en algún programa de rehabilitación. De los 110 que sí cumplieron, 62 (56.4\%) participantes abandonaron el programa de tratamiento en diferentes momentos del desarrollo del mismo, con lo cual solo fueron 48 (43.6\%) participantes los que finalizaron el programa. Es importante indicar que este nivel de deserción o abandono de los participantes en programas de tratamiento para agresores, incluso con la obligatoriedad de la orden judicial, es muy común en los diferentes contextos en los que se realizan (Echauri, 2010; Conchell, 2011; Catalá, 2014; Ocampo, 2018). Sobre este aspecto se discutirá en el apartado correspondiente. Por otro lado, siguiendo la clasificación propuesta por Amor et al., (2009) se trata de agresores de bajo y moderado riesgo.

\section{Instrumentos}

Escala de Tácticas de Conflictos (CTS-2): se utilizó La CTS-2 en su adaptación al español de Loinaz (2009) basada en la original de Straus et al., (1996). Consta 
de un total de 78 ítems, es decir, 39 ítems dobles, uno para cada miembro de la pareja. La CTS-2 contiene cinco subescalas, cada una de las cuales se divide a su vez en dos subescalas. La subescala de negociación está dividida en cognitiva y emocional, y las otras cuatro subescalas (agresiones psicológicas, agresiones físicas, coerción sexual y daños) en leve y severa. Para este estudio sólo se han utilizado los ítems de estas cuatro y específicamente las que corresponden para la víctima, ya que se ha considerado evaluar el cambio a partir de la información que ella provea pues los agresores muy a menudo intentan presentar una imagen favorable de sí mismos, con altos niveles de deseabilidad social (Echeburúa, Amor y Corral, 2003). Su consistencia interna (alfa de Cronbach) varía según la subescala y la muestra, pero oscila entre .34 y .94 (Straus, 2004), con propiedades similares en las versiones en inglés y español. Straus $(1996,2004)$ en su versión original informaba coeficientes de confiabilidad de .53 a .91 , posteriormente informó la utilización de la escala en 17 naciones con un promedio de .70 a .81 de correlación interna del instrumento.

Inventario de Pensamientos Distorsionados (IPMV): Ferrer et al., (2006) analizaron las propiedades psicométricas del IPMV originalmente desarrollado por Echeburúa y Fernández-Montalvo (1997), aplicándolo a una muestra de 1395 estudiantes. Modificaron el sistema de respuesta, sustituyendo la respuesta dicotómica Sí/No, por una escala Likert de cuatro puntos ( 1 completamente en desacuerdo, 4 completamente de acuerdo). El coeficiente alpha de Cronbach inicial fue de .81 (consistencia interna del test). Excepto para los ítems 8, 19, 27, 28 y 29 , que fueron eliminados, las correlaciones de cada ítem con la puntuación total eran mayores a .30, reduciéndose así la escala a 24 ítems y obteniendo un alpha de .84. Por último, los autores propusieron una solución del inventario en cuatro factores, que explicaban el $46,46 \%$ de la varianza total: la subescala 1 ( 7 ítems) evalúa la aceptación del estereotipo tradicional y la misoginia ("una mujer no debe contradecir a su marido", "cuando un hombre le pega a su mujer es porque algún motivo debe haber"), la subescala 2 (8 ítems) evalúa la culpabilización de las mujeres víctimas del maltrato ("muchas mujeres provocan a propósito a sus maridos para que estos pierdan el control y las golpeen", "si una mujer que ha sido maltratada no pide ayuda o no hace la denuncia es porque quiere proteger a su marido"), la subescala 3 (5 ítems) evalúa la aceptación de la violencia como forma adecuada para resolver los conflictos ("las cachetadas a veces son necesarias", "lo que ocurre en una familia es problema únicamente de la familia"), y por último la subescala 4 (4 ítems) analiza la minimización de la violencia contra las mujeres y la des-culpabilización del agresor ("muy pocas mujeres tienen huellas físicas o psicológicas a causa de los malos tratos", "una mujer que permanece conviviendo con un hombre violento debe tener un serio problema psicológico").

- Escala de Atribución de Responsabilidad y Minimización (IRM): Elaborada por Lila et al., (2008), es una escala de doce ítems para medir, en 
primer lugar, dónde sitúan los agresores contra su pareja la culpa de la situación que los llevó a ser sentenciados (los tres primeros factores) y, en segundo término, el nivel en que le quitan importancia a los hechos por los que han sido sentenciados. Se les solicita a los participantes que señalen su grado de acuerdo con las afirmaciones planteadas en los ítems en una escala de 1 a $5(1=$ totalmente en desacuerdo; $5=$ totalmente de acuerdo). La escala presenta 4 dimensiones:

1. Culpabilización de la víctima, esta dimensión está compuesta de tres ítems que evalúa el nivel en que el individuo le atribuye la culpa de su situación a las mentiras y/ características de personalidad o conducta de la víctima $(\alpha=.73)$ ("estoy aqui debido a las mentiras y exageraciones de mi pareja").

2. Defensa propia, compuesta de dos ítems que miden el nivel en que el sujeto sostiene que su conducta se ha producido únicamente como respuesta a una agresión previa $(\alpha=.79)$ ("estoy aqui por haberme defendido de las agresiones de mi pareja").

3. Autoatribución de culpa, tres ítems que evalúan el nivel en que el individuo atribuye la causa de los hechos a problemas personales o a su propia forma de $\operatorname{ser}(\alpha=.70)$ ("mis celos son la causa de que me encuentre en esta situación").

4. Minimización, está compuesta por cuatro ítems que evalúan el nivel en que le quitan importancia a los hechos por los que han sido sentenciados $(\alpha=.68)$ ("la causa de que esté aqui es que se llama 'violencia contra la pareja' a cualquier cosa").

\section{Programa de tratamiento}

Se trata de un programa para agresores de corte cognitivo-conductual introduciendo aspectos relacionados con la perspectiva de género (Ruiz et al., 2010; De los Galanes y Tabernero, 2013; Pérez et al., 2013; Menacho et al., 2015; Alarcón, 2020). Es un programa progresivo que busca inicialmente la detención de la conducta violenta, hasta, en su parte final, que el participante aprenda habilidades de comunicación y solución de conflictos (la Tabla 1 presenta la estructura del programa). Consta de 32 sesiones grupales, efectuándose con una frecuencia de una sesión semanal de dos horas de duración (120 minutos). El programa se caracteriza por tres aspectos diferenciales: a) integra aspectos clínicos con perspectiva de género, b) señala la necesidad de trabajar la motivación inicial de los agresores, y c) hace un análisis de las diversas conductas que configuran la violencia de género. Para considerar cumplido el programa se consideró más del 75\% (24 sesiones como mínimo) de asistencias al mismo, registradas a través de la lista de asistencia. 
Tabla 1

Estructura del programa de tratamiento

\begin{tabular}{|c|c|c|}
\hline Módulo & Contenido del tratamiento & Técnicas terapéuticas \\
\hline \multicolumn{3}{|c|}{ Objetivo: responsabilización de los abusos cometidos } \\
\hline \multirow[t]{4}{*}{ Principios básicos } & $\begin{array}{l}\text { Concepto de violencia y rueda de expe- } \\
\text { riencias personales ( } 2 \text { sesiones) }\end{array}$ & \multirow{4}{*}{$\begin{array}{l}\text { Exposición psicoeducativa } \\
\text { Reestructuración cognitiva }\end{array}$} \\
\hline & Tipos de violencia (2 sesiones) & \\
\hline & El ciclo de la violencia & \\
\hline & La escalada de la violencia & \\
\hline Asunción de responsabilidad & $\begin{array}{l}\text { Asunción de responsabilidad ( } 2 \\
\text { sesiones) }\end{array}$ & $\begin{array}{l}\text { Exposición psicoeducativa } \\
\text { Modelamiento }\end{array}$ \\
\hline \multicolumn{3}{|c|}{ Objetivo: control del maltrato y eliminación de distorsiones cognitivas } \\
\hline $\begin{array}{l}\text { Identificación y expresión } \\
\text { emocional }\end{array}$ & $\begin{array}{l}\text { Identificación y expresión emocional } \\
\text { (2 sesiones) }\end{array}$ & $\begin{array}{l}\text { Modelamiento } \\
\text { Retroalimentación }\end{array}$ \\
\hline Distorsiones cognitivas & $\begin{array}{l}\text { Creencias irracionales y pensamientos } \\
\text { distorsionados sobre los roles de género } \\
(3 \text { sesiones) }\end{array}$ & Reestructuración cognitiva \\
\hline \multirow[t]{3}{*}{$\begin{array}{l}\text { Estrategias para la detención } \\
\text { de la violencia }\end{array}$} & $\begin{array}{l}\text { Análisis funcional de la conducta ( } 2 \\
\text { sesiones) }\end{array}$ & \multirow{3}{*}{$\begin{array}{l}\text { Suspensión temporal } \\
\text { Distracción cognitiva } \\
\text { Entrenamiento en autoinstrucciones }\end{array}$} \\
\hline & Control de la ira (2 sesiones) & \\
\hline & Relajación & \\
\hline \multicolumn{3}{|c|}{ Objetivo: prevención de factores precipitantes directos } \\
\hline Género y violencia de género & $\begin{array}{l}\text { Violencia de género ( } 2 \text { sesiones) } \\
\text { Sexualidad masculina }\end{array}$ & $\begin{array}{l}\text { Exposición psicoeducativa } \\
\text { Reestructuración cognitiva }\end{array}$ \\
\hline Violencia en la familia & $\begin{array}{l}\text { Violencia en la familia de origen } \\
\text { Estilos parentales ( } 2 \text { sesiones })\end{array}$ & $\begin{array}{l}\text { Reestructuración cognitiva } \\
\text { Exposición imaginaria }\end{array}$ \\
\hline
\end{tabular}

Objetivo: desarrollo de habilidades de comunicación

\begin{tabular}{lll}
\hline $\begin{array}{l}\text { Entrenamiento en habilidades } \\
\text { interpersonales }\end{array}$ & Asertividad (2 sesiones) & \\
& Empatía & $\begin{array}{l}\text { Entrenamiento asertivo } \\
\text { Entrenamiento en negociación } \\
\text { Entrenamiento en solución de conflictos }\end{array}$ \\
& Negociación (2 sesiones) & \\
& Solución de conflictos (2 sesiones) & \\
Prevención de recaídas & Prevención de recaídas & $\begin{array}{l}\text { Ensayo conductual } \\
\text { Retroalimentación }\end{array}$
\end{tabular}

Nota: Adaptado de Ruiz et al., (2010).

Asimismo, en investigaciones donde se evalúa la eficacia de programas de tratamiento para agresores contra su pareja la conformación de grupos de tratamiento y grupos de control no suele ser fácil pues hay que tener en cuenta los criterios éticos y la distribución aleatoria quedaría condicionada, entre otros temas, por la gravedad del maltrato, la duración de la sanción, el riesgo de reincidencia, el grado de voluntariedad de los participantes y la disponibilidad de recursos terapéuticos. Por estas razones, la mayoría de las veces se utiliza diseños cuasi 
experimentales (Edleson \& Syers, 1990; Saunders, 1996; Gondolf, 1999) cuyos objetivos principales están relacionados con la evaluación del cambio actitudinal respecto de la violencia de género (rol de la mujer, justificación de la violencia, atribución de la responsabilidad a la pareja, etc.), la evaluación de la reincidencia de comportamientos de maltrato (datos provistos por la policía o poder judicial, e información directa de la pareja del agresor) y por la efectividad de los programas de intervención según su duración.

Así, en este estudio no se ha conformado un grupo de control sin tratamiento o de lista de espera, primero, porque el curso de las conductas de maltrato suele ser crónico y de larga duración. Segundo, porque resulta muy difícil mantener la motivación de este tipo de participantes para aceptar evaluaciones repetidas sin recibir ninguna intervención terapéutica. Y tercero, porque la investigación se ha llevado a cabo en el marco de los compromisos que la institución que facilito el acceso a la muestra mantiene con el sistema judicial, lo que hizo imposible el mantenimiento de los participantes en un grupo de control sin tratamiento.

\section{Procedimiento}

Se realizó las coordinaciones necesarias para la realización del estudio con las instituciones de salud a las que los juzgados de familia remitían directamente a los participantes para su atención. Cada nuevo participante que se incluía en el programa de tratamiento, en su primera sesión (pudo ampliarse a más sesiones cuando así se requirió), participaba de una evaluación para la verificación de los criterios de inclusión y la firma del compromiso de participación, además se especificaban las normas del programa, las actividades y las obligaciones, y finalmente de una entrevista y la primera evaluación con los cuestionarios y test estandarizados mencionados. Asimismo, a aquellos participantes que cumplían con los criterios de inclusión, se les pidió información de contacto de la víctima (su pareja o ex pareja) para que el responsable del estudio se comunicara con ella y se evaluara el riesgo de ser nuevamente víctima de violencia, además de solicitarle también su consentimiento informado ya que se le requirió que proporcione información sobre la conducta violenta del participante. Esto fue así porque, como ya se ha señalado con anterioridad, los hombres agresores contra su pareja y que son obligados judicialmente a llevar cabo una terapia presentan altos niveles de deseabilidad social (Echeburúa, Amor y Corral, 2003).

En vista de la heterogeneidad de la muestra, principalmente en relación a su formación académica, el tiempo necesario para completar todos los instrumentos de evaluación fue variable, entre 90 y 120 minutos. La evaluación inicial de cada uno se hizo de forma individual por psicólogos entrenados para la evaluación e intervención psicológica de agresores. Se buscó que el participante sea lo más sincero posible. 
Cuando un participante finalizaba su proceso de tratamiento, se llevaba a cabo una última sesión de evaluación postratamiento en la que se realizaba una entrevista final, además de la segunda aplicación de los cuestionarios de forma individual. Asimismo, se realizaba una entrevista final con la víctima para poder evaluar la disminución o detención de la conducta violenta del participante.

\section{Análisis de datos}

Para el análisis de los datos se usó el SPSS v.24. En tanto finalizaron por completo el tratamiento menos de 50 casos, se realizó el test de Shapiro-Wilk para contrastar la distribución normal de los datos. Habiendo demostrado que las variables se distribuyen en forma normal, se utilizó un contraste paramétrico, la prueba $t$ para muestras relacionadas ya que se trata de una misma muestra evaluada en dos momentos distintos. Y en consonancia a las exigencias y estándares internacionales vigentes, se utilizó la medida del tamaño del efecto paramétrico Delta de Glass (Domínguez; 2013).

\section{RESULTADOS}

En la Tabla 2 se puede observar que la media de las variables en el postratamiento ha cambiado en la dirección esperada, vale decir, han disminuido la frecuencia de agresiones psicológicas, agresiones físicas, coerción sexual y daños. Del mismo modo, como puede verse en la Tabla 3, de acuerdo al nivel de significación, dicho cambio en cada una de las variables ha sido significativo. Con relación al tamaño del efecto, calculado mediante la Delta de Glass, este es grande (Morales-Vallejo, 2012).

\section{Tabla 2}

Estadisticos descriptivos pre y post de las dimensiones de la conducta violenta (tácticas de conflicto) de los agresores en su relación de pareja

\begin{tabular}{lccc}
\hline & Media & $\boldsymbol{N}$ & $\boldsymbol{D} \boldsymbol{T}$ \\
\hline Agresiones psicológicas T1 & 8.1875 & 48 & 4.94504 \\
Agresiones psicológicas T2 & 2.0208 & 48 & 2.51370 \\
Agresiones físicas T1 & 3.6667 & 48 & 3.05505 \\
Agresiones físicas T2 & .2708 & 48 & 1.04657 \\
Coerción sexual T1 & .8542 & 48 & 1.36817 \\
Coerción sexual T2 & .2708 & 48 & .67602 \\
Daños T1 & .3542 & 48 & .75764 \\
Daños T2 & .0208 & 48 & .14434 \\
\hline
\end{tabular}

Nota: $\mathrm{N}=$ Número de participantes; DT = Desviación Estándar 
Tabla 3

Prueba t (muestras relacionadas) y tamaño del efecto de las dimensiones de la conducta violenta (tácticas de conflicto) de los agresores en su relación de pareja

\begin{tabular}{lcccccc}
\hline & \multicolumn{2}{c}{$95 \%$ IC } & & & \\
\cline { 2 - 7 } & Inferior & Superior & $\boldsymbol{t}$ & $\boldsymbol{g l}$ & $\boldsymbol{p}$ & $\boldsymbol{d}$ \\
\hline Agr. psicológicas T1 & 5.04234 & 7.29100 & 11.034 & 47 & .000 & 2.453 \\
Agr. psicológicas T2 & & & & & & \\
Agresiones físicas T1 & & & & & & \\
Agresiones físicas T2 & 2.54506 & 4.24660 & 8.030 & 47 & .000 & 3.244 \\
Coerción sexual T1 & .28484 & .88183 & 3.931 & 47 & .000 & .862 \\
Coerción sexual T2 & & & & & & \\
Daños T1 & .10656 & .56011 & 2.957 & 47 & .005 & 2.309 \\
Daños T2 & & & & & & \\
\hline
\end{tabular}

Nota: $t=\mathrm{t}$ de student; $g l=$ grados de libertad $p=<.01 ; d=$ delta de glass; $\mathrm{N}=48$.

En la Tabla 4 se puede observar que la media de las variables en el postratamiento ha cambiado en la dirección esperada, es decir, en el grupo de agresores contra su pareja han disminuido sus distorsiones cognitivas relacionados con la mujer y el uso de la violencia (misoginia, culpabilización de la víctima, aceptación de la violencia como forma adecuada de resolver problemas y minimización de la violencia). Del mismo modo, como puede verse en la Tabla 5, de acuerdo al nivel de significación, dicho cambio en cada una de las variables ha sido significativo. Con relación al tamaño del efecto, calculado mediante la Delta de Glass, este es grande (Morales-Vallejo, 2012).

\section{Tabla 4}

Estadísticos descriptivos pre y post de las distorsiones cognitivas relacionadas con la mujer y el uso de la violencia

\begin{tabular}{lccc}
\hline & Media & $\boldsymbol{N}$ & $\boldsymbol{D} \boldsymbol{T}$ \\
\hline Misoginia T1 & 13.4167 & 48 & 3.51188 \\
Misoginia T2 & 8.7917 & 48 & 2.86548 \\
Culpabilización T1 & 15.6250 & 48 & 3.08479 \\
Culpabilización T2 & 10.2708 & 48 & 2.95166 \\
Acep. violencia T1 & 10.1458 & 48 & 2.25963 \\
Acep. violencia T2 & 6.8542 & 48 & 1.85644 \\
Minimización T1 & 15.1042 & 48 & 2.74629 \\
Minimización T2 & 10.9375 & 48 & 2.40041 \\
\hline
\end{tabular}

Nota: $\mathrm{N}=$ Número de participantes; DT = Desviación Estándar 
Tabla 5

Prueba t (muestras relacionadas) y tamaño del efecto de las distorsiones cognitivas relacionadas con la mujer y el uso de la violencia

\begin{tabular}{lcccccc}
\hline & \multicolumn{7}{c}{ 95\% IC } & & & & \\
\cline { 2 - 7 } & Inferior & Superior & $\boldsymbol{t}$ & $\boldsymbol{g l}$ & $\boldsymbol{p}$ & $\boldsymbol{d}$ \\
\hline Misoginia T1 & 3.83346 & 5.41654 & 11.755 & 47 & .000 & 1.614 \\
Misoginia T2 & & & & & & \\
Culpabilización T1 & 4.30211 & 6.40622 & 10.238 & 47 & .000 & 1.813 \\
Culpabilización T2 & & & & & & \\
Acep. violencia T1 & 2.50164 & 4.08169 & 8.382 & 47 & .000 & 1.773 \\
Acep. violencia T2 & & & & & & \\
Minimización T1 & 3.25958 & 5.07376 & 9.241 & 47 & .000 & 1.735 \\
Minimización T2 & & & & & & \\
\hline
\end{tabular}

Nota: $t=\mathrm{t}$ de student; $g l=$ grados de libertad; $p=<.01 ; d=$ delta de glass; $\mathrm{N}=48$.

En la Tabla 6 se observa que la media de las variables en el postratamiento se ha modificado en la dirección esperada, es decir, ha existido un cambio en el nivel en el que el participante coloca la culpa de su situación en las mentiras y/o características de personalidad de la víctima (culpabilización), en el nivel en que asevera que su conducta violenta se ha producido únicamente en respuesta a una agresión previa (defensa propia) y en el nivel en que le quitan importancia a los hechos por los que han sido demandados (minimización). Asimismo, como puede verse en la Tabla 7, de acuerdo al nivel de significación, dicho cambio en cada una de las variables ha sido significativo. Con relación al tamaño del efecto, calculado mediante la Delta de Glass, este es grande (Morales-Vallejo, 2012).

\section{Tabla 6}

Estadísticos descriptivos pre y post de los componentes de la atribución de responsabilidad y minimización

\begin{tabular}{llll}
\hline & Media & N & DT \\
\hline Culpabilización T1 & 13.1042 & 48 & 1.78933 \\
Culpabilización T2 & 6.5625 & 48 & 3.19512 \\
Defensa propia T1 & 6.9167 & 48 & 3.06548 \\
Defensa propia T2 & 3.3750 & 48 & 2.31209 \\
Minimización T1 & 7.0000 & 48 & 3.16228 \\
Minimización T2 & 4.3125 & 48 & 2.02281 \\
\hline
\end{tabular}

Nota: $\mathrm{N}$ = Número de participantes; DT = Desviación Estándar 
Tabla 7

Prueba t (muestras relacionadas) y tamaño del efecto de los factores de la atribución de responsabilidad y minimización

\begin{tabular}{|c|c|c|c|c|c|c|}
\hline & \multicolumn{2}{|c|}{$95 \%$ IC } & \multirow[b]{2}{*}{$T$} & \multirow[b]{2}{*}{$g l$} & \multirow[b]{2}{*}{$p$} & \multirow[b]{2}{*}{$D$} \\
\hline & Inferior & Superior & & & & \\
\hline $\begin{array}{l}\text { Culpabilización T1 } \\
\text { Culpabilización T2 }\end{array}$ & 5.66756 & 7.41577 & 15.056 & 47 & .000 & 2.047 \\
\hline $\begin{array}{l}\text { Defensa propia } \mathrm{T} 1 \\
\text { Defensa propia } \mathrm{T} 2\end{array}$ & 2.42434 & 4.65900 & 6.377 & 47 & .000 & 1.531 \\
\hline $\begin{array}{l}\text { Minimización T1 } \\
\text { Minimización T2 }\end{array}$ & 1.88353 & 3.49147 & 6.725 & 47 & .000 & 1.328 \\
\hline
\end{tabular}

Nota: $t=\mathrm{t}$ de student; $g l=$ grados de libertad; $p=<.01 ; d=$ delta de glass; $\mathrm{N}=48$.

\section{DISCUSIÓN}

El programa del tratamiento cognitivo conductual ha sido efectivo para reducir la conducta violenta en un grupo de agresores en su relación de pareja, lo cual va en la línea de estudios realizados por Pérez et al., 2013; Echauri et al., 2013; Boira et al., 2013; Arrigoni et al., 2013; De los Galanes y Tabernero, 2013; Conchell et al., 2016; Carbajosa et al., 2017; Fernández-Montalvo et al., 2019; Vargas et al., 2020, Fernández-Montalvo et al., 2020. Además, al igual que en los estudios de Gondolf (1997, 2004), una parte importante de los datos para evaluar la eficacia han partido de informes facilitados por las mujeres víctimas de la violencia, teniendo en cuenta que el objetivo principal en todas las intervenciones de este tipo es la reducción de la conducta violenta del agresor y las otras razones ya mencionadas (Echeburúa, Amor y Corral, 2003). De igual manera, los datos de este trabajo coinciden con los obtenidos por otros investigadores que colocan la no reincidencia de la conducta violenta entre un $50 \%$ y un $80 \%$, basándose en la información proporcionada por las propias mujeres (Ruíz et al., 2010).

Siendo más específicos, en la conducta violenta se han distinguido las agresiones psicológicas, agresiones físicas, coerción sexual y daños. Se ha comprobado que el programa ha sido efectivo para reducir los diferentes tipos de conductas violentas de los agresores. Estos resultados son consistentes con lo hallado por Redondo (2012), quien encontró disminuciones significativas en las diferentes conductas violentas en varios grupos de agresores tras haber recibido tratamiento. Estos resultados indicarían que las intervenciones psicológicas generan cambios significativos en la reducción de la conducta violenta; no obstante, siendo el presente un estudio sin grupo control, queda pendiente de seguir comprobándolo en futuras investigaciones peruanas.

En cuanto a la disminución de las distorsiones cognitivas sobre con la mujer y el uso de la violencia, los resultados de este estudio concuerdan con los hallazgos 
de Boira et al. (2013), Arrigoni et al. (2013) y Fernández-Montalvo et al. (2019). Una modificación sustancial de estructuras profundas del pensamiento, como son las distorsiones cognitivas solo se puede lograr con programas terapéuticos extensos, intensivos y continuados (Martínez y Pérez, 2009), un aspecto que también ha sido señalado por Echeburúa y Fernández-Montalvo (2009), quienes refieren que la modificación de actitudes machistas y violentas suele darse como resultado de un largo sobreaprendizaje. Esta es una de las razones por las que se ha propuesto una intervención razonablemente larga (alrededor de 9 meses), sumada a las sugerencias de Hamberger \& Hastings (1988), Faulkner et al (1992) y Gondolf (1999), quienes encontraron que reducciones significativas en la realización de conductas graves de maltrato y la modificación de variables asociadas al maltrato, se daban luego de haber pasado por un tratamiento extenso en comparación con uno de corta duración. Además, se ha encontrado que el número de sesiones de intervención grupal logra un efecto negativo en la reincidencia, es decir, a mayor número de sesiones, menor será la probabilidad de reincidir (Morales et al., 2012). Por otro lado, Boira et al. (2013) encontraron que al comparar los resultados entre la modalidad de terapia grupal abierta (donde los terapeutas decidieron el área de trabajo de cada sesión en función de la evolución del grupo) versus la modalidad de terapia grupal estructurada (donde las áreas de trabajo se abordaron siguiendo un orden estructurado en función de la secuencia marcada en el programa), la terapia grupal estructurada ha mostrado valores de cambio más significativos. Coincidiendo con ellos, un programa estructurado, como el propuesto aquí, ha demostrado ser idóneo para lograr disminuir las variables relacionadas con el maltrato.

Respecto a las atribuciones de responsabilidad de los agresores, esperando que se produzcan más atribuciones relacionadas con la asunción de responsabilidad por sus propios actos y menos atribuciones que sitúen la culpa en terceras personas (la víctima) o agentes externos (la ley, la sociedad, el alcohol, etc.), los resultados, que son congruentes con lo hallado por Conchell et al. (2012), Pérez et al. (2013), Conchell et al. (2016) y Vargas et al. (2020), permiten afirmar que la intervención ha producido cambios en todas las atribuciones. Es decir, en primer lugar, ha habido una reducción en la culpabilización a la víctima, en segundo lugar, en el nivel en que el participante expresa que su conducta se ha producido únicamente como respuesta a una agresión previa (defensa propia) y, por último, en la actitud de restar importancia a los hechos por los que ha sido sentenciado (minimización). Todo esto supondría que el participante luego de la intervención llega a tomar conciencia de uno de los supuestos básicos necesarios para el cambio: que él tiene una importante responsabilidad en esta situación que le ha llevado a ser enjuiciado (Conchell, 2011). Así, la intervención habría buscado lograr una genuina asunción de responsabilidad, vale decir, que cada participante se haga consciente de su situación: dónde está, qué es lo que le ha llevado aquí y que la mejora de su conducta es posible únicamente desde el momento en que asuma que ello depende de él mismo. De hecho, aumentar la asunción de responsabilidad y 
disminuir la culpabilización a la víctima es uno de los principales objetivos de la intervención con maltratadores (Lila et al., 2008; Catalá, 2014). Esto tiene relevancia puesto que el hecho de que empiecen a asumir su responsabilidad sobre su conducta violenta, será un importante factor protector y un punto clave de motivación para el cambio. Logro relevante puesto que se trata de participantes obligados judicialmente a llevar a cabo un tratamiento psicológico. Por ello, considerando las altas tasas de abandono que en general se han encontrado en programas de este tipo en diversos contextos (Echauri, 2010; Conchell, 2011; Catalá, 2014), uno de los principales factores a trabajar dentro del marco terapéutico es el tema de la motivación para el cambio. La falta de reconocimiento del problema por parte del agresor o la adopción de una postura de autosuficiencia, con un supuesto control de la situación, obstaculizan la búsqueda de ayuda terapéutica. Aunque el temor a la pérdida de su pareja e hijos y la visión de un futuro en soledad, así como el temor a las repercusiones legales y a un detrimento de capacidad adquisitiva, pueden actuar como un acicate, no terminan siendo factores de una motivación apropiada, manteniendo un compromiso del cambio fluctuante (Dutton y Golant, 1997). Además, aun considerando que el sistema judicial (como factor coercitivo) tiene un importante valor para el logro de los objetivos de un programa de intervención ya que las personas que son obligadas judicialmente tienen un $16 \%$ más de probabilidad de completar un programa de tratamiento que aquellos que participan por otras instancias (Morales et al., 2012), se tiene por consenso la necesidad de enfatizar en el establecimiento de una relación terapéutica asentada en la confianza y confidencialidad que permitan en el participante una motivación auténtica para el cambio de conducta. En ese sentido, siguiendo el modelo del cambio de conducta propuesto por Prochaska \& DiClemente (1983), se ha encontrado, al igual que en otras investigaciones (De los Galanes y Tabernero, 2013), que los resultados muestran a participantes evolucionando desde estadios de precontemplación hacia estadios de actuación y mantenimiento a lo largo de la terapia, considerando además que en esas últimas etapas de cambio los participantes presentan una mayor probabilidad de desarrollar conductas de empatía y comunicación asertiva.

Todos estos resultados permiten concluir que el programa de intervención estudiado logra disminuir las conductas violentas e incide positivamente en las variables relacionadas con la violencia sobre la pareja.

\section{CONCLUSIONES}

- El programa del tratamiento mostró ser eficaz en la disminución de la conducta violenta en un grupo de agresores en su relación de pareja. Específicamente, ha logrado reducir significativamente la frecuencia de agresiones psicológicas, de agresiones físicas, de coerciones sexuales y de daños sobre la víctima. 
- El programa de tratamiento logró disminuir de forma eficaz las distorsiones cognitivas relacionadas con la mujer y el uso de la violencia en un grupo de agresores en su relación de pareja. Específicamente, ha logrado reducir significativamente el nivel de misoginia, de culpabilización de la víctima, de aceptación de la violencia como forma adecuada de resolver problemas y de minimización de la violencia.

- El programa de tratamiento logró ser eficaz para modificar las atribuciones de responsabilidad de su conducta violenta en un grupo de agresores en su relación de pareja. Específicamente, ha logrado modificar significativamente el nivel en el que el participante coloca la culpa de su situación en supuestas mentiras y/o características de personalidad de la víctima (culpabilización), el nivel en el que expresa que su conducta violenta se ha producido únicamente como respuesta a un maltrato previo (defensa propia) y en el nivel en que le resta importancia a los hechos por los que ha sido demandado (minimización).

- El programa de tratamiento ha mostrado unos resultados claramente esperanzadores y podría ser considerado como una intervención eficaz para la disminución o detención de la violencia contra la pareja. No obstante, de la misma forma que ocurre con la evaluación de programas en otros ámbitos de intervención, los resultados pueden ser limitados debido sobre todo a imperativos éticos que devienen en limitaciones metodológicas en nuestra carrera por lo que serían necesarias más investigaciones antes de hacer aseveraciones definitivas al respecto.

\section{Fuentes de financiamiento / Funding:}

Recursos propios.

\section{Rol de los autores / Authors Roles:}

FLV: Investigador y autor principal del estudio.

AQP: Co-autor del artículo y coordinador del acceso a la muestra.

\section{Aspectos éticos / legales; Ethics / legals:}

Hemos cumplido las normas éticas de investigación contando con las autorizaciones y consentimientos informados los cuales tenemos bajo resguardo.

\section{Conflicto de intereses / Competing interests:}

Declaramos que no hemos tenido conflicto de intereses. 


\section{REFERENCIAS}

Alarcón, B. (2020). Eficacia de los programas especificos de violencia de género y seguridad vial como medidas penales alternativas. [Tesis Doctoral, Universidad de Murcia]. https://igitum.um.es/digitum/handle/10201/101465

Amor, P.; Echeburúa, E. y Loinaz, I. (2009). ¿Se puede establecer una clasificación tipológica de los hombres violentos contra su pareja? International Journal Of Clinical and Health Psychology, 9(3), 519-539. https://www.redalyc.org/articulo. oa? $\mathrm{id}=33712038010$

Arrigoni, F.; Jiménez, J.; Navarro, J. y Mendoza, P. (2013). Aplicación de un programa terapéutico en hombres violentos contra la pareja. Anuario de Psicología Jurídica. 23, 3-9. https://doi.org/10.5093/aj2013a2

Ato, M., López-García, J. J., \& Benavente, A. (2013). Un sistema de clasificación de los diseños de investigación en psicología. Anales de Psicología / Annals of Psychology, 29(3), 1038-1059. https://doi.org/10.6018/analesps.29.3.178511

Boira, S.; López, Y.; Tomás-Aragonés, L. y Gaspar, A. (2013). Intervención psicológica en la comunidad en hombres condenados por violencia de género. Anales de Psicología, 29(1), 19-28. http://dx.doi.org/10.6018/analesps.29.1.130631

Carbajosa, P.; Catalá, A.; Lila, M. y Gracia, E. (2017). Differences in treatment adherence, program completion, and recidivism among batterer subtypes. The European Journal of Psychology Applied to Legal Context, 9(2), 93-101. https://dx.doi.org/10.1016/j. ejpal.2017.04.001

Catalá, A. (2014). Explorando nuevas vías para mejorar la eficacia de la intervención en hombres condenados por violencia de género. [Tesis Doctoral, Universidad de Valencia]. https://roderic.uv.es/handle/10550/35018

Conchell, R. (2011). La intervención en medio abierto con hombres penados por violencia contra la mujer: Un análisis pre-post de los indicadores de eficacia del Programa Contexto. [Tesis Doctoral, Universidad de Valencia]. https://roderic.uv.es/handle/10550/38706

Conchell, R.; Lila, M. y Catalá, A. (2012). Cambios psicosociales en un programa de intervención con hombres penados por violencia contra la mujer. Revista de Psicología, 2l(2), 159-186. https://www.redalyc.org/articulo.oa?id=26424861007

Conchell, R.; Lila, M. y Catalá, A. (2016). Intervención con hombres penados por violencia contra la mujer en las relaciones de pareja: el Programa Contexto. Información Psicológica, 111, 51-63. http://dx.medra.org/10.14635/IPSIC.2016.111.2

Congreso de la República (2015, 23 de noviembre). Ley $N^{\circ} 30364$ para prevenir sancionar y erradicar la violencia contra las mujeres y los integrantes del grupo familiar. Diario Oficial El Peruano $\mathrm{N}^{\circ}$ 14636. https://bit.ly/3q8T6IM

De los Galanes, M. y Tabernero, C. (2013). El impacto del entrenamiento cognitivo-conductual. Un estudio exploratorio con agresores de género. Anuario de Psicología Jurídica, 23, 11-19. https://doi.org/10.5093/aj2013a3 
Deza, S. y Guzmán, M. (2009). Intervención psicoeducativa con hombres que ejercen violencia - HEV: El modelo multidimensional. Revista Avances en Psicología Volumen, 17(1), 75-83. http://revistas.unife.edu.pe/index.php/avancesenpsicologia/article/ view/1909

Domínguez, S. (2013). Tamaño del efecto: Cálculo mediante un módulo en Visual Basic. Revista de Investigación en Psicología, 16(2), 235-240. https://doi.org/10.15381/rinvp. v16i2.6556

Dutton, D. y Golant, S. (1997). El golpeador. Paidós.

Echauri, J. (2010). Eficacia de un programa de tratamiento psicológico para hombres maltratadores en la relación de pareja. Características psicopatológicas y resultados terapéuticos. [Tesis doctoral no publicada, Universidad Pública de Navarra].

Echauri, J.; Fernández-Montalvo, J.; Martínez, M. y Azkarate, J. (2013). Effectiveness of a treatment programme for inmigrants who committed gender-based violence against their partner. Psicothema, 25(1), 49-54. DOI: 10.7334/psicothema2012.75

Echeburúa, E. y Fernández-Montalvo, J. (1997). Tratamiento cognitivo conductual de hombres violentos en el hogar: un estudio piloto. Análisis y Modificación de Conducta, 23(89), 355-384. https://dialnet.unirioja.es/servlet/articulo?codigo=7069996

Echeburúa, E., Amor, P.J. y Corral, P. (2002). Mujeres maltratadas en convivencia prolongada con el agresor: variables relevantes. Acción Psicológica, 1(2), 135-150. http://espacio.uned.es/fez/view/bibliuned:AccionPsicologica2002-numero2-0003

Echeburúa, E.; Amor, P. y Corral, P. (2003). Autoinformes y entrevistas en el ámbito de la psicología clínica forense. Limitaciones y nuevas perspectivas. Análisis y Modificación de Conducta, 29(126), 503-522. https://dialnet.unirioja.es/servlet/ articulo?codigo $=761627$

Echeburúa, E.; Fernández-Montalvo, J. y Amor, P. (2003). Psychopatological profile of men convicted of gender violence. A study in the prisons of Spain. Journal of Interpersonal Violence, 18(7), 798-812. https://doi.org/10.1177/0886260503253300

Echeburúa, E. y Fernández-Montalvo, J. (2009). Evaluación de un programa de tratamiento en prisión de hombres condenados por violencia grave contra la pareja. International Journal of Clinical and Health Psychology, 9(1), 5-20. https://www.redalyc.org/ articulo.oa? $\mathrm{id}=33712020001$

Echeburúa, E. y Redondo, S. (2010). ¿Por qué víctima es femenino y agresor masculino? Pirámide.

Edleson, J. L., \& Syers, M. (1990). Relative effectiveness of group treatments for men who batter. Social Work Research \& Abstracts, 26(2), 10-17. https://doi.org/10.1093/ swra/26.2.10

INEI (2019). Encuesta Demográfica y de Salud Familiar (ENDES). https://www.inei.gob. pe/media/MenuRecursivo/publicaciones_digitales/Est/Endes2019/

Faulkner, K.; Stoltemberg, C.; Cogen, R.; Nolder, M. \& Shooter, E. (1992). Cognitivebehavioral group treatment for male spouse abusers. Journal of Family Violence, 7(1), 37- 55. https://doi.org/10.1007/BF00978723 
Fernández-Montalvo, J.; Echauri, J.; Martínez, M. y Azcárate, J. (2011). Violencia de género e inmigración: perfil diferencial de hombres maltratadores nacionales e inmigrantes. Behavioral Psychology / Psicología Conductual, 19(2), 439-452. https://bit. ly/38xK $2 \mathrm{~Hz}$

Fernández-Montalvo, J.; Arteaga, A.; y López-Goñi, J. J. (2019). Treatment effectiveness of intimate partner violence perpetration among patients in a drug addiction program. Psychology of Violence, 9(2), 156-166. https://doi.org/10.1037/vio0000184

Fernández-Montalvo, J., Echauri, J.A., Siria, S., López-Goñi, J.J., Azcárate, J.M. y Martínez, M. (2020). Is psychological treatment equally effective for intimate partner violence perpetrators with and without childhood family violence? Legal and Criminological Psychology. https://doi.org/10.1111/lcrp.12187

Ferrer, V.; Bosch, E.; Ramis, M.; Torres, E. y Navarro, C. (2006). La violencia contra las mujeres en la pareja: creencias y actitudes en estudiantes universitarios. Psicothema, 18, 359-366. http://www.psicothema.com/pdf/3223.pdf

García-Moreno, C.; Jansen, H.; Ellsberg, M.; Heise, L. \& Watts, C. (2005). WHO Multicountry study on women's health and domestic violence against women. World Health Organization. https://apps.who.int/iris/handle/10665/43310

Gondolf, E. (1997). Batterer Programmes: What we know and what we need to know. Journal of Interpersonal Violence, 12(1), 83-98. https://doi.org/10.1177/088626097012001006

Gondolf, E. (1999). A comparison of four batterer interventions systems: Do court referral, program length, and services matter? Journal of Interpersonal Violence, 14(1), 41-61. https://doi.org/10.1177/088626099014001003

Gondolf, E. (2004). Evaluating batterer counseling programs: A difficult task showing some effects and implications. Aggression and Violent Behavior, 9(6), 605-631. https://doi.org/10.1016/j.avb.2003.06.001

Hamberger, L. \& Hasting, J. (1988). Skills training for treatment of spouse abusers: an outcome study. Journal of Family Violence, 3(2), 121-130. https://doi.org/10.1007/ BF00994029

Kaufman, M. (1997). Las experiencias contradictorias del poder entre los hombres. En T. Valdés y J. Olavarría (eds.). Masculinidades. Poder y Crisis (pp. 63-81). Isis Internacional/FLACSO Chile.

Lila, M., Herrero, J. \& Gracia, E. (2008). Evaluating attribution of responsibility and minimization by male batterers: Implications for batterer programs. The Open Criminology Journal, 1, 4-11. http://dx.doi.org/10.2174/1874917800801010004

Loinaz, I. (2009). Aproximación teórica y empírica al estudio de las tipologías de agresores. Análisis descriptivo de variables e instrumentos de evaluación en el centro penitenciario Brians-2. Ministerio del Interior-Secretaría General Técnica. https:// eige.europa.eu/library/resource/imio.C00047098c

Martínez, M. y Pérez, M. (2009). Evaluación criminológica y psicológica de los agresores domésticos. Centre d' Estudis Jurídics i Formació Especialitzada: Generalitat de 
Cataluña. https://www.comunidad.madrid/sites/default/files/doc/justicia/evaluacin_criminol-gica_y_psicol-gica_de_los_agresores.pdf

Medina, J. (2002). Violencia contra la mujer en la pareja: investigación comparada y situación en España. Tirant lo Blanch.

Menacho, I.; Marchena, E.; Navarro, J. y Vinaza, I. (2015). Intervención en hombres condenados por violencia de género. International Journal of Developmental and Educational Psychology, 2(1), 405-417. http://dx.doi.org/10.17060/ijodaep.2015.n1.v2.135

Menéndez, S.; Pérez, J. y Lorence, B. (2013). La violencia de pareja contra la mujer en España: Cuantificación y caracterización del problema, las víctimas, los agresores y el contexto social y profesional. Psychosocial Intervention, 22(1), 41-53. http://dx.doi. org/10.5093/in2013a6

Morales-Vallejo, P. (2012). El tamaño del efecto (effect size): Análisis complementarios al contraste de medias. https://web.upcomillas.es/personal/peter/investigacion/ Tama\%floDelEfecto.pdf

Ocampo, I. (2018). Hombres que han ejercido violencia intrafamiliar: la deserción en un programa de intervención municipal en Montevideo, Uruguay. GÉNEROS - Multidisciplinary Journal of Gender Studies, 7(3), 1762-1785. https://hipatiapress.com/ hpjournals/index.php/generos/article/view/3709/2586

OMS (2013). Estimaciones mundiales y regionales de la violencia contra la mujer. Prevalencia y efectos de la violencia conyugal y de la violencia sexual no conyugal en la salud. https://apps.who.int/iris/handle/10665/85243

OMS (2016, 29 de noviembre). Violencia contra la mujer. https://www.who.int/es/newsroom/fact-sheets/detail/violence-against-women

Morales, A.; Muñoz, N.; Trujillo, M.; Hurtado, M.; Cárcamo, J. y Torres, J. (2012) Los programas de intervención con hombres que ejercen violencia contra su pareja mujer. Fundamentos teórico-criminológicos. Evidencia internacional de su efectividad y evaluación de impacto de un programa en Chile. Paz Ciudadana y Gendarmería. https://biblio.dpp.cl/contenido/16017\%20

Pérez, M.; Giménez-Salinas, A. y Juan, M. (2013). Evaluación de la eficacia del programa de tratamiento con agresores de pareja (PRIA) en la comunidad. Psychosocial Intervention, 22(2), 105-114. http://dx.doi.org/10.5093/in2013a13

Prochaska, J. \& DiClemente, C. (1983). Stages and proceses of selfchange of smoking: Toward and integrative model of change. Journal of Consulting and Clinical Psychology, 51(3), 390-395. https://doi.org/10.1037/0022-006X.51.3.390

Redondo, N. (2012). Eficacia de un programa de tratamiento psicológico para maltratadores. [Tesis Doctoral, Universidad Complutense de Madrid]. https://eprints.ucm.es/ id/eprint/15003/

Ruiz, S.; Negredo, L.; Ruiz, A.; García-Moreno, C.; Herrero, O.; Yela, M. y Pérez- Ramírez, M. (2010). Violencia de género: Programa de intervención para agresores (PRIA). Secretaría General Técnica (Ministerio del Interior). https://bit.ly/3kBov5H 
Saunders, D. (1996). Feminist-cognitive-behavioral and process-psychodynamic treatments for men who batter: Interaction of abuser traits and treatment models. Violence and Victims, 11(4), 393-413. https://pubmed.ncbi.nlm.nih.gov/9210279/

Seidler, V. (2000). La sinrazón masculina. Paidós.

Straus, M.; Hamby, S.; Boney-McCoy, S. \& Sugarman, D. (1996). The revised conflicto tactics scale: Development and preliminary psychometric data. Journal of Family Issues, 17(3), 283-316. https://doi.org/10.1177/019251396017003001

Straus, M. (2004). Cross-cultural reliability and validity of the Revised Conflict Tactics Scales: A study of university student dating couples in 17 nations. Cross-Cultural Research: The Journal of Comparative Social Science, 38(4), 407-432. https://doi. org/10.1177/1069397104269543

Vargas, V., Conchell, R., Expósito-Álvarez, C., y Lila, M. (2020). Diferencias entre latinoamericanos y españoles participando en un programa de intervención en violencia de género: Resultados proximales y finales. Anales De Psicología / Annals of Psychology, 36(3), 418-426. https://doi.org/10.6018/analesps.428831 
